\title{
Remodelling Metabolic Syndrome Components to Adapt them to the Tunisian Context and to Determine a Sole Metric Criterion
}

\author{
Sarraj Mohamed Youssef ${ }^{1 *}$, Najah Mohamed ${ }^{1}$, Slimani Afef ${ }^{1}$, Ben Hamda Khaldoun ${ }^{3}$, Neffati Fadoua ${ }^{2}$, Najjar Mohamed Fadhel ${ }^{2}$ and Slimane \\ Mohamed Naceur ${ }^{1}$ \\ ${ }^{1}$ Research Unit genetic and biological factors of atherosclerosis. Medicine Faculty, University of Monastir, Tunisia \\ ${ }^{2}$ Laboratory of Biochemistry and Toxicology of the Fatouma Bourguiba University Hospital of Monastir, Tunisia \\ ${ }^{3}$ Department of Cardiology of the Fatouma Bourguiba University Hospital of Monastir, Tunisia
}

\begin{abstract}
Aims: Metabolic Syndrome (MS) is a heterogenic entity and its major limitation was the use of binary components and the dependence of some criteria to ethnic origin and/or gender. We seek to remodel MS criteria to adapt them to the Tunisian context and to determine a sole quantitative MS parameter.

Materials and methods: 592 subjects who underwent routine control were investigated for biochemical, anthropometric and clinical examination. The diagnosis of MS was based on the IDF and AHA/NHLBI definition. The computer model HOMA 2 was used to determine HOMA- $\beta$, HOMA-S and HOMA-IR. Triglycerides (TG), High Density Lipoprotein cholesterol (cHDL), Systolic Blood Pressure (SBP), HOMA-IR and Waist Circumference Stature (WCS) were used to calculate the different MS markers. The area under curve of ROC curves were used to compare the powers of these MS parameters.
\end{abstract}

Results: HOMA-IR was more informative about glycaemia abnormality, WCS express better android obesity, and TG/cHDL ratio characterizes dyslipidemia. (TG $x$ WCS $\times$ HOMA-IR $\times$ SBP) / cHDL parameter was more accurate to estimate MS occurrence; its cut-off point is 106.75

Conclusion: This marker, with sensitivity and specificity of 94.4 and 85.1 per cent, can be used either to diagnose or to predict MS occurrence.

Keywords: Metabolic syndrome; Metabolic syndrome criteria cutoff values; Sole metric tool; Tunisian context

\section{Introduction}

Epidemiological studies have suggested an increased prevalence of metabolic syndrome (MS) in Tunisian population $[1,2]$.

MS was originally introduced by Reaven as syndrome X or insulin resistance syndrome, is recognized clinically by the presence of three or more of these components; abdominal obesity, elevated Triglycerides (TG), low High Density Lipoprotein cholesterol (cHDL), elevated Blood Pressure (BP) and high Fasting Plasma Glucose (FPG).

MS is the concurrence of multiple metabolic abnormalities associated with the development and progression of atherosclerosis [3]. Meta-analyses found that the MS increases the risk of cardiovascular disease and mortality [4-6].

Moreover, MS can be considered as an independent risk factor for male hypogonadism [7]. In addition, all components of the MS have individually been associated with the incidence and progression of chronic kidney diseases [8].

Environmental factors such as low physical activity and inappropriate dietary habits are strong determinants of the MS. Moreover, genetic factors also contribute to the individual susceptibility to MS [9].

MS is a heterogenic entity and its major limitation was the use of binary components and some criteria depending on ethnic group and/ or sex. Moreover, there was a mosaique of combinations of three to five criteria.

Our approach was to remodel MS criteria to adapt them to the Tunisian context and to determine a sole metric parameter combining MS quantitative components to be used as a screening tool either to diagnose or to predict MS occurrence.

\section{Materials and Methods}

\section{Study population}

592 subjects who underwent routine control were investigated for biochemical, anthropometric and clinical examination at the department of occupational medicine of the University Hospital of Monastir, Tunisia. Participants gave their written informed consent prior to their participation. The study was approved by the ethical committee of the Hospital.

\section{Diagnostic criteria for metabolic syndrome}

The diagnosis of MS was based on the IDF and AHA/NHLBI definition, which requires the presence of at least three of the following criteria: the central (abdominal) obesity (defined as Waist Circumference (WC) $\geq 94 \mathrm{~cm}$ in men and $\geq 80 \mathrm{~cm}$ in women), the raised TG $\geq 1.70 \mathrm{mmol} / \mathrm{L}$ (drug treatment for elevated triglycerides is an alternate indicator), the reduced $\mathrm{cHDL}<1.04 \mathrm{mmol} / \mathrm{L}$ in men and $<1.29$ $\mathrm{mmol} / \mathrm{L}$ in women (or specific treatment for this lipid abnormality), the elevated Systolic Blood Pressure (SBP) $\geq 130 \mathrm{mmHg}$ and/or Diastolic Blood Pressure (DBP) $\geq 85 \mathrm{mmHg}$ (antihypertensive drug treatment in a patient with a history of hypertension was an alternate indicator),

*Corresponding author: Sarraj Mohamed Youssef, Research Unit 05/UR/09 12: Genetic and Biological Factors of Atherosclerosis, Medicine Faculty University of Monastir, Tunisia, Tel: 216-73-462 200; Fax: 216-73-460-737; E-mail: Youssef_sarraj@yahoo.fr

Received April 28, 2013; Accepted May 13, 2013; Published May 15, 2013

Citation: Youssef SM, Mohamed N, Afef S, Khaldoun BH, Fadoua N, et al. (2013) Remodelling Metabolic Syndrome Components to Adapt them to the Tunisian Context and to Determine a Sole Metric Criterion. J Metabolic Synd 2: 121. doi:10.4172/2167-0943.1000121

Copyright: (c) 2013 Youssef SM, et al. This is an open-access article distributed under the terms of the Creative Commons Attribution License, which permits unrestricted use, distribution, and reproduction in any medium, provided the original author and source are credited. 
Citation: Youssef SM, Mohamed N, Afef S, Khaldoun BH, Fadoua N, et al. (2013) Remodelling Metabolic Syndrome Components to Adapt them to the Tunisian Context and to Determine a Sole Metric Criterion. J Metabolic Synd 2: 121. doi:10.4172/2167-0943.1000121

Page 2 of 4

and the elevated FPG $\geq 5.56 \mathrm{mmol} / \mathrm{L}$ or previously diagnosed type 2 diabetes [10].

\section{Anthropometric measurements}

Height and weight were measured according to a standardized protocol in the study population, with subjects wearing light clothing and no shoes. Body Mass Index (BMI) was calculated by dividing weight in kilograms by height in square meters $\left(\mathrm{kg} / \mathrm{m}^{2}\right)$. The Waist Circumference (WC) was measured in the horizontal plane at the midpoint between the lowest rib and the iliac crest. The Waist Circumference Stature (WCS) was calculated by dividing [WC $(\mathrm{cm})]$ by height $(\mathrm{cm})$. Systolic Blood Pressure (SBP) and Diastolic Blood Pressure (DBP) were measured to the nearest $5 \mathrm{mmHg}$ with a mercury sphygmomanometer, with subjects in a supine position and having relaxed for 5 minutes.

\section{Biochemical analysis}

The blood samples of the study population were collected in the morning after a 12-hour fasting period, heparinized plasma was immediately obtained by blood centrifugation at $4^{\circ} \mathrm{C}$ at $3000 \mathrm{rpm}$ for $15 \mathrm{~min}$. All analyses were carried out on Cobas $6000^{\mathrm{TM}}$ analyzer (Roche Diagnostics Mannheim, Germany), in biochemistry and toxicology laboratory of the Hospital. Serum Triglycerides (TG), serum High Density Lipoprotein cholesterol (cHDL) and Fasting Plasma Glucose (FPG) were measured by enzymatic methods. Fasting plasma insulin (FPI) was measured by Electrochemiluninescence Immuno Assay (ECLIA).

The computer model HOMA 2 was used to determine $\beta$-cell function (HOMA- $\beta \%$ ), insulin sensitivity (HOMA-S \%), and Insulin Resistance (HOMA-IR) from paired fasting glucose (mmol/L) and insulin (mIU/L) concentrations [11].

\section{Statistical analysis}

Data were analyzed by SPSS 17.0 for Windows. Continuous

\begin{tabular}{|c|c|c|c|}
\hline Variables & MS- $(n=484)$ & $\mathrm{MS}^{+}(n=108)$ & $p$ \\
\hline Age (years) & $41.2(29.1-51.7)$ & $38.4(29.4-53.6)$ & 0.705 \\
\hline Gender M/ F (\%) & $241 / 243(49.8 / 50.2)$ & $54 / 54(50.0 / 50.0)$ & 0.969 \\
\hline Diabetes [n (\%)] & $46(9.5)$ & $30(27.8)$ & $<0.001$ \\
\hline Hypertension [n (\%)] & $66(13.6)$ & $43(39.8)$ & $<0.001$ \\
\hline $\mathrm{SBP}(\mathrm{mmHg})$ & $120(115-125)$ & $140(120-140)$ & $<0.001$ \\
\hline $\mathrm{DBP}(\mathrm{mmHg})$ & $80(70-80)$ & $80(80-90)$ & $<0.001$ \\
\hline $\mathrm{BMI}\left(\mathrm{kg} / \mathrm{m}^{2}\right)$ & $24.5(23.4-25.8)$ & $27.9(26.8-29.6)$ & $<0.001$ \\
\hline Men WC $(\mathrm{cm})$ & $93(89-95)$ & $98(96-101)$ & $<0.001$ \\
\hline Women WC $(\mathrm{cm})$ & $79(77-86)$ & $93(90-98)$ & $<0.001$ \\
\hline WCS & $0.52(0.49-0.54)$ & $0.58(0.56-0.60)$ & $<0.001$ \\
\hline $\mathrm{TG}(\mathrm{mmol} / \mathrm{L})$ & $0.95(0.71-1.26)$ & $1.93(1.51-2.48)$ & $<0.001$ \\
\hline Men cHDL (mmol/L) & $1.10(1.02-1.16)$ & $0.84(0.74-0.92)$ & $<0.001$ \\
\hline Women cHDL (mmol/L) & $1.45(1.34-1.53)$ & $1.10(0.89-1.26)$ & $<0.001$ \\
\hline FPG (mmol/L) & $4.80(4.49-5.14)$ & $6.14(4.89-8.00)$ & $<0.001$ \\
\hline $\mathrm{FPI}(\mathrm{mlU} / \mathrm{L})$ & $7.39(6.58-9.06)$ & $12.20(9.54-13.13)$ & $<0.001$ \\
\hline HOMA- $\beta \%$ & $102(86-123)$ & $84(55-111)$ & $<0.001$ \\
\hline HOMA-S\% & $105(85-118)$ & $58(53-81)$ & $<0.001$ \\
\hline HOMA-IR & $0.9(0.8-1.2)$ & $1.7(1.2-1.9)$ & $<0.001$ \\
\hline
\end{tabular}

MS: Without Metabolic Syndrome; MS ${ }^{+}$: With Metabolic Syndrome; SBP: Systolic Blood Pressure; DBP: Diastolic Blood Pressure: BMI: Body Mass Index; WC: Waist Circumference; WCS: Waist Circumference Stature; TG: Triglycerides; cHDL: High Density Lipoprotein -Cholesterol; FPG; Fasting Plasma Glucose; FPI; Fasting Plasma Insulin; HOMA- $\beta \%$ : \% $\beta$ Cell Function; HOMA-S\%: \%Cell Insulin Sensitivity; HOMA-IR: Insulin Resistance

Table 1: Anthropometric and biochemical characteristics of study population.

\begin{tabular}{|c|c|c|c|c|c|}
\hline MS Variables & AUC $(95 \%$ C I) & $p$ & $\begin{array}{l}\text { Cut-off } \\
\text { point }\end{array}$ & $\begin{array}{c}\% \\
\text { Sensitivity }\end{array}$ & $\begin{array}{c}\% \\
\text { Specificity }\end{array}$ \\
\hline \multicolumn{6}{|c|}{ Impaired glycaemia criteria } \\
\hline FPG (mmol/L) & $0.760(0.698-0.823)$ & $<0.001$ & 5.14 & 70.4 & 74.6 \\
\hline FPI (mlU/L) & $0.798(0.749-0.847)$ & $<0.001$ & 9.71 & 75.0 & 79.1 \\
\hline HOMA- $\beta \%$ & $0.669(0.604-0.734)$ & $<0.001$ & 96 & 60.7 & 63.9 \\
\hline HOMA-S\% & $0.814(0.763-0.865)$ & $<0.001$ & 84 & 76.4 & 75.9 \\
\hline HOMA-IR & $0.817(0.766-0.867)$ & $<0.001$ & 1.2 & 79.6 & 78.9 \\
\hline \multicolumn{6}{|l|}{ Obesity criteria } \\
\hline $\mathrm{BMI}\left(\mathrm{kg} / \mathrm{m}^{2}\right)$ & $0.864(0.824-0.904)$ & $<0.001$ & 25.9 & 87.0 & 75.4 \\
\hline WC (cm) & $0.803(0.749-0.857)$ & $<0.001$ & 91.5 & 80.6 & 69.2 \\
\hline WCS & $0.875(0.837-0.913)$ & $<0.001$ & 0.54 & 88.0 & 71.9 \\
\hline \multicolumn{6}{|c|}{ Dyslipidemia criteria } \\
\hline $\mathrm{cHDL}(\mathrm{mmol} / \mathrm{L})$ & $0.830(0.785-0.875)$ & $<0.001$ & 1.04 & 80.4 & 75.0 \\
\hline TG (mmol/L) & $0.877(0.841-0.912)$ & $<0.001$ & 1.21 & 84.3 & 70.5 \\
\hline TG/ cHDL & $0.908(0.878-0.938)$ & $<0.001$ & 1.24 & 88.0 & 78.7 \\
\hline \multicolumn{6}{|c|}{ Hypertension criteria } \\
\hline SBP $(\mathrm{mmHg})$ & $0.825(0.773-0.877)$ & $<0.001$ & 125 & 74.1 & 82.4 \\
\hline $\mathrm{DBP}(\mathrm{mmHg})$ & $0.704(0.640-0.767)$ & $<0.001$ & 80 & 77.8 & 35.5 \\
\hline $\begin{array}{l}\text { SBPxDBP } \\
(\mathrm{mmHg})^{2}\end{array}$ & $0.775(0.714-0.837)$ & $<0.001$ & 9487 & 76.9 & 37.0 \\
\hline $\begin{array}{l}\text { SBP+DBP } \\
(\mathrm{mmHg})\end{array}$ & $0.783(0.724-0.843)$ & $<0.001$ & 198 & 79.6 & 36.4 \\
\hline $\begin{array}{l}\mathrm{SBP}+\mathrm{DBP} / 2 \\
(\mathrm{mmHg})\end{array}$ & $0.783(0.724-0.843)$ & $<0.001$ & 99 & 79.6 & 36.4 \\
\hline
\end{tabular}

MS: Metabolic Syndrome; AUC: Area Under Curve; CI: Confidence Interval; FPG Fasting Plasma Glucose; FPI: Fasting Plasma Insulin; HOMA- $\beta \%$ : $\% \beta$ Cell Function; HOMA-S\%: \%Cell Insulin Sensitivity; HOMA-IR: Insulin Resistance; BMI: Body Mass Index; WC: Waist Circumference; WCS: Waist Circumference Stature cHDL: High Density Lipoprotein Cholesterol; TG: Triglycerides; SBP: Systolic Blood Pressure; DBP: Diastolic Blood Pressure

Table 2: Receiver Operating Characteristics (ROC) curves analysis to establish the best metabolic syndrome components cut-off points.

results are expressed as median and quartile; and frequencies for qualitative variables. Comparisons among groups were assessed using the independent-sample $t$ test for quantitative variables and Pearson's chi-square test for qualitative variables. The area under curve (AUC) of the Receiver Operating Characteristic (ROC) curves was used for predicting a better marker for MS incidence. ROC curve analysis was employed to select the best cut-off points of each marker which have the highest value for MS occurrence. The pair index (1-specificity, sensitivity) was used to determine optimal cut-off points. A two-sided $p<0.05$ was considered as statically significant.

\section{Results}

Among the 592 subjects who were enrolled, 108 presented metabolic syndrome $\left(\mathrm{MS}^{+}\right)$and 484 were without $\left(\mathrm{MS}^{-}\right)$. The prevalence rate of MS in our study population was $18.2 \%$.

Table 1 shows that $\mathrm{MS}^{+}$subjects have higher BMI, WC, WCS, SBP, DBP, TG, FPG and FPI; and reduced cHDL compared to subjects MSWe also noted elevated IR and reduced HOMA $\beta$ and HOMA S in MS group.

Table 2 shows that the largest AUC were obtained with HOMA-IR, followed by HOMA-S, indicating that the model with HOMA-IR was superior in estimating MS impaired glycaemia.

WCS express better android obesity independently of gender. TG/ cHDL ratio characterizes dyslipidemia. SBP was more informative about hypertension.

Table 3 shows that each parameter was a significant discriminating 
Citation: Youssef SM, Mohamed N, Afef S, Khaldoun BH, Fadoua N, et al. (2013) Remodelling Metabolic Syndrome Components to Adapt them to the Tunisian Context and to Determine a Sole Metric Criterion. J Metabolic Synd 2: 121. doi:10.4172/2167-0943.1000121

Page 3 of 4

\begin{tabular}{|c|c|c|c|c|c|}
\hline MS Parameters & AUC $(95 \% \mathrm{C} \mathrm{I})$ & $p$ & Cut-off point & $\%$ Sensitivity & $\%$ Specificity \\
\hline TG/cHDL & $0.908(0.878-0.938)$ & $<0.001$ & 1.24 & 88.0 & 78.7 \\
\hline$(\mathrm{TG} \times \mathrm{WCS}) / \mathrm{cHDL}$ & $0.926(0.900-0.952)$ & $<0.001$ & 0.60 & 90.7 & 75.6 \\
\hline$(T G \times$ WCS $\times$ HOMA-IR) / cHDL & $0.942(0.923-0.961)$ & $<0.001$ & 0.87 & 92.6 & 84.5 \\
\hline$(T G \times$ WCS $\times$ HOMA-IR $\times$ SBP) / cHDL & $0.956(0.940-0.973)$ & $<0.001$ & 106.75 & 94.4 & 85.1 \\
\hline
\end{tabular}

MS: Metabolic Syndrome; AUC: Area Under Curve; Cl: Confidence Interval; TG: Triglycerides; cHDL: High Density Lipoprotein Cholesterol; WCS: Waist Circumference Stature; HOMA-IR: Insulin Resistance; SBP: Systolic Blood Pressure

Table 3: Receiver Operating Characteristics (ROC) curves analysis to establish the best metabolic syndrome parameter cut-off points.

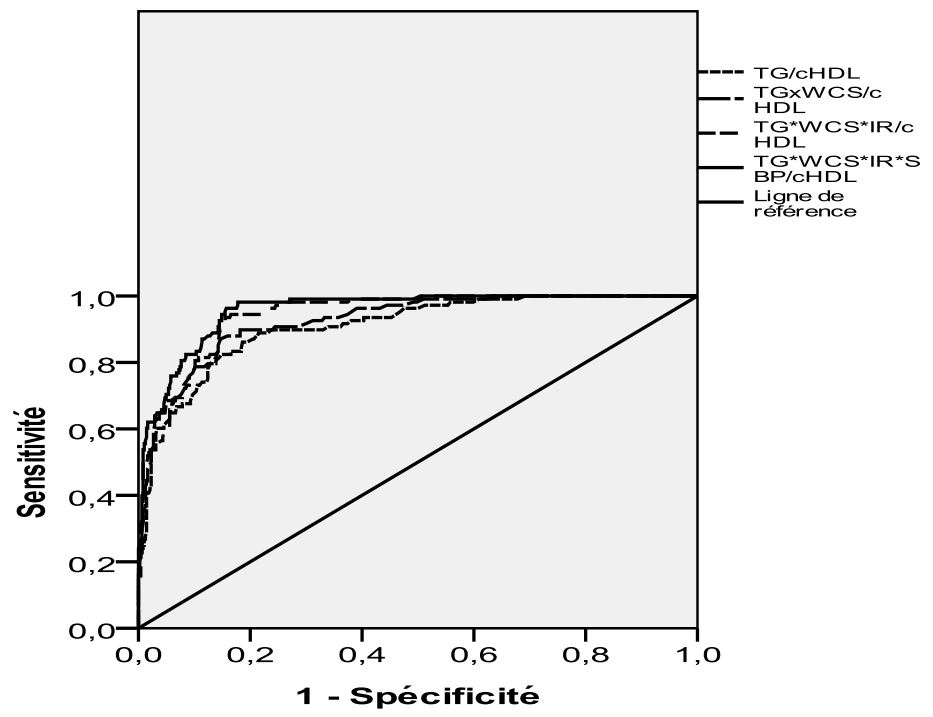

Figure 1: ROC curves of MS parameters for MS occurrence.

factor for MS and there was a significant stepwise increase in AUC of parameters with each additional quantitative MS criterion in estimating MS occurrence. The largest AUC was obtained with (TG $x$ WCS $x$ HOMA-IR x SBP) / cHDL marker indicating that this new parameter was the estimated MS occurrence (Figure 1). Its cut-off point was 106.75 .

The optimal cut-off point for prediction of MS incidence in present study was with sensitivity and specificity of 94.4 and 85.1 per cent.

\section{Discussion}

A pragmatic approach was required to overcome the paradox of the importance of MS in public health and the practical problems of its diagnosis. The fact that the five variables are used as "present" or "absent" likely makes these screening tools less than perfect for the optimal diagnosis of the MS. Moreover, there were 243 combinations possible of MS components. The central issue was to determinate a sole combining quantitative MS parameter to use to predict MS incidence.

Glucose is the main item in MS. The balance between glucose production and utilization is regulated by a network of hormones, neural pathways, and metabolic signals. Insulin plays a pivotal role in this process. As blood glucose levels rise, impaired glucose tolerance and then type 2 diabetes develops. Thus, there is a continuous spectrum of insulin responsiveness, ranging from normal insulin sensitivity to severe IR [12]. Regarding the TG/cHDL ratio, the major quantitative change is associated with the insulin resistance $[13,14]$ and this ratio is independent of the sex. The degree of impairment of glucose metabolism is influenced both by the insulin sensitivity of cells within the body and by pancreatic $\beta$-cell reserve capacity [15]. Furthermore, the end of insulin sensitivity is the beginning of insulin resistance. However, to establish a sole MS specific criterion, we multiplied this ratio by WCS, which was more accurate to estimating MS occurrence than WC or BMI. In addition, waist to stature ratio is more strongly associated with cardiovascular risk factors than other simple anthropometric indices [16].

Many studies demonstrated the relationship between IR, obesity, and dyslipidemia [17-19]. Moreover, we multiplied this previous equation by HOMA-IR, who was superior in estimating impaired glycaemia and by SBP that express better hypertension in MS.

This new parameter (TG $x$ WCS $x$ HOMA-IR $x$ SBP)/cHDL, was more accurate marker to estimating MS occurrence.

In fact, this metric tool, with sensitivity $=94.4$ and specificity $=85.1$ per cent can be used either to diagnose or to predict MS incidence.

To confirm our funding, another analysis ought to be conducted in Tunisia.

\section{Conclusion}

In Tunisian context, a sole quantitative parameter combining quantitative metabolic syndrome criteria may be used as a screening tool to diagnose MS which is accompanied by a higher cardiovascular risk.

\section{References}

1. Ezzaher A, Haj MD, Mechri A, Neffati F, Douki W, et al. (2011) Metabolic syndrome in Tunisian bipolar I patients. Afr Health Sci 11: 414-420.

2. Boumaiza I, Omezzine A, Rejeb J, Rebhi L, Rejeb NB, et al. (2011) Association 
Citation: Youssef SM, Mohamed N, Afef S, Khaldoun BH, Fadoua N, et al. (2013) Remodelling Metabolic Syndrome Components to Adapt them to the Tunisian Context and to Determine a Sole Metric Criterion. J Metabolic Synd 2: 121. doi:10.4172/2167-0943.1000121

between eight adiponectin polymorphisms, obesity, and metabolic syndrome parameters in Tunisian volunteers. Metab Syndr Relat Disord 9: 419-426.

3. Reaven GM (1988) Banting lecture 1988. Role of insulin resistance in human disease. Diabetes 37: 1595-1607.

4. Ford ES (2005) Risks for all-cause mortality, cardiovascular disease, and diabetes associated with the metabolic syndrome: a summary of the evidence. Diabetes Care 28: 1769-1778.

5. Galassi A, Reynolds K, He J (2006) Metabolic syndrome and risk of cardiovascular disease: a meta-analysis. Am J Med 119: 812-819.

6. Gami AS, Witt BJ, Howard DE, Erwin PJ, Gami LA, et al. (2007) Metabolic syndrome and risk of incident cardiovascular events and death: a systematic review and meta-analysis of longitudinal studies. J Am Coll Cardiol 49: 403414.

7. Buvat J, Maggi M, Gooren L, Guay AT, Kaufman J, et al. (2010) Endocrine aspects of male sexual dysfunctions. J Sex Med 7: 1627-1656.

8. Chen J, Muntner P, Hamm LL, Jones DW, Batuman V, et al. (2004) The metabolic syndrome and chronic kidney disease in U.S. adults. Ann Intern Med 140: $167-174$

9. Liese AD, Mayer-Davis EJ, Tyroler HA, Davis CE, Keil U, et al. (1997) Familial components of the multiple metabolic syndrome: the ARIC study. Diabetologia 40: $963-970$

10. Alberti KG, Eckel RH, Grundy SM, Zimmet PZ, Cleeman JI, et al. (2009) Harmonizing the metabolic syndrome: a joint interim statement of the International Diabetes Federation Task Force on Epidemiology and Prevention; National Heart, Lung, and Blood Institute; American Heart Association; World
Heart Federation; International Atherosclerosis Society; and International Association for the Study of Obesity. Circulation 120: 1640-1645.

11. Wallace TM, Levy JC, Matthews DR (2004) Use and abuse of HOMA modeling Diabetes Care 27: 1487-1495.

12. Abrahamson MJ (2007) A 74-year-old woman with diabetes. JAMA 297: 196204

13. Taskinen MR (2003) Diabetic dyslipidaemia: from basic research to clinical practice. Diabetologia 46: 733-749.

14. Tangvarasittichai S, Poonsub P, Tangvarasittichai O (2010) Association of serum lipoprotein ratios with insulin resistance in type 2 diabetes mellitus. Indian J Med Res 131: 641-648.

15. Miranda PJ, DeFronzo RA, Califf RM, Guyton JR (2005) Metabolic syndrome: definition, pathophysiology, and mechanisms. Am Heart J 149: 33-45.

16. Ho SY, Lam TH, Janus ED; Hong Kong Cardiovascular Risk Factor Prevalence Study Steering Committee (2003) Waist to stature ratio is more strongly associated with cardiovascular risk factors than other simple anthropometric indices. Ann Epidemiol 13: 683-691.

17. Després JP (2006) Intra-abdominal obesity: an untreated risk factor for Type 2 diabetes and cardiovascular disease. J Endocrinol Invest 29: 77-82.

18. Després JP (2001) Health consequences of visceral obesity. Ann Med 33: 534 541.

19. Bauduceau B, Vachey E, Mayaudon H, Burnat P, Dupuy O, et al. (2007) Should we have more definitions of metabolic syndrome or simply take wais measurement? Diabetes Metab 33: 333-339. 